\title{
Éditorial
}

\section{L'antibioprophylaxie de l'endocardite infectieuse en pratique bucco-dentaire : recherche Vérité désespérément !}

\author{
Philippe Lesclous
}

Un article récemment paru dans la célèbre revue britannique The Lancet vient de relancer le débat sur ce sujet [1]. Dans cette publication, Mark Dayer et ses collègues rapportent les résultats d'une importante étude épidémiologique basée sur des données hospitalières britanniques portant sur les périodes 2004-2013 pour l'antibioprophylaxie (ABP) et 20002013 pour les endocardites infectieuses (EI). Ils montrent une petite mais significative augmentation de l'incidence des EI depuis 2008. Cette augmentation est objectivée chez les sujets à haut risque d'EI mais également chez les sujets à risque plus faible. Concomitamment, les auteurs soulignent une diminution très nette, de presque $90 \%$, de l'ABP de l'EI en pratique bucco-dentaire. Le parallèle entre ces deux phénomènes est bien sûr tentant et relance de manière forte le débat sur la place de l'ABP dans la prévention de l'EI. Une lecture rapide et superficielle de ce travail peut introduire la confusion chez les patients comme chez les praticiens.

Pour troublants qu'ils soient, ces résultats ne doivent pas conduire à des conclusions hâtives. Bien que l'ABP systématique ne soit plus recommandée en Grande-Bretagne, même chez les sujets à haut risque d'EI, depuis mars 2008 par le NICE (National Institute for health and Clinical Excellence, l'équivalent britannique de la Haute Autorité de Santé française) par manque de preuve d'efficacité, les résultats de la présente étude ne fournissent aucune corrélation au sens statistique du terme. En effet, il s'agit d'une étude observationnelle, non randomisée, dont quelques points clés méritent d'être soigneusement discutés. D'abord et indépendamment de toute $A B P$, le vieillissement général de la population britannique pendant cette période et l'augmentation afférente des cardiopathies à risque d'EI (quel que soit le degré de sévérité) ainsi que des facteurs de comorbidité à potentiel infectieux important comme le diabète par exemple ne sont nullement pris en compte. De plus, le diagnostic d'EI d'origine bucco-dentaire apparaît probabiliste puisque non étayé par « une preuve microbiologique ». Si l'augmentation de cette incidence était imputable à la chute de l'ABP, elle ne devrait être visible que chez les sujets ayant une EI à streptocoques oraux et non dans les autres sous-groupes. Autre point, le nombre d'actes buccodentaires invasifs qui auraient pu nécessiter une ABP (avant son arrêt en 2008) est très mal renseigné. Il est aussi intéressant de noter que ces résultats viennent contredire ceux enregistrés par la même équipe dans une étude de suivi trois ans après l'interruption de l'ABP publiée en 2011 [2] dans laquelle aucune augmentation significative de l'incidence de l'EI n'était rapportée. Enfin, il semble que de sérieuses réserves soient à formuler sur l'analyse statistique menée dans cette étude.

D'ailleurs, Dayer et ses collègues doivent être salués car eux-mêmes ne concluent pas à une corrélation entre ces deux phénomènes, tout simplement parce que cette étude, aussi riche soit-elle, ne le permet pas.

Il est aussi important de souligner que, dans le reste du monde, malgré l'instauration d'une ABP systématique dont le champ d'application s'est réduit aux seuls patients à haut risque d'EI ces dernières années, l'incidence et la mortalité associée à l'endocardite infectieuse n'ont pas baissé et restent inchangées, en particulier en France [3].

Ces deux approches et les résultats qu'elles génèrent illustrent parfaitement que l'absence de preuve de l'efficacité d'une telle procédure n'est pas la preuve de l'absence de son efficacité. Toutes deux reconnaissent bien l'existence d'EI à streptocoques oraux.

Il est aussi important de signaler que les deux approches promeuvent au plus haut point les mesures antiseptiques locales (au premier rang desquelles l'hygiène orale) de manière à minimiser l'importance des bactériémies dites spontanées lors des activités quotidiennes.

\section{Alors, où se trouve la vérité ?}

Il y a urgence à mettre en œuvre une étude randomisée, contrôlée, internationale pour évaluer l'efficacité réelle de

\footnotetext{
*Correspondance : philippe.lesclous@univ-nantes.fr
} 
l'ABP dans la prévention de l'EI. Cette étude, pour difficile qu'elle soit à concevoir étant donné les comportements prophylactiques différents, est vraiment indispensable. Nous la devons à nos patients.

\section{Références}

1. Dayer MJ, Jones S, Prendergast B, Baddour LM, Lockhart PB, Thornhill MH. Incidence of infective endocarditis in England, 2000-13: a secular trend, interrupted time-series analysis.
Lancet 2014 http://dx.doi.org/10.1016/S0140-6736(14)62007-9. [Epub ahead of print]

2. Thornhill MH, Dayer MJ, Forde JM, et al. Impact of the NICE guideline recommending cessation of antibiotic prophylaxis for prevention of infective endocarditis: before and after study. BMJ 2011:342:d2392.

3. Duval X, Delahaye F, Alla $F$ et al. Temporal trends in infective endocarditis in the context of prophylaxis guideline modifications: three successive population-based surveys. J Am Coll Cardiol 2012;59:1968-76. 